\title{
Interference Alignment in Dense Wireless Networks
}

\author{
Urs Niesen
}

\begin{abstract}
We consider arbitrary dense wireless networks, in which $n$ nodes are placed in an arbitrary (deterministic) manner on a square region of unit area and communicate with each other over Gaussian fading channels. We provide inner and outer bounds for the $n \times n$-dimensional unicast and the $n \times 2^{n}$-dimensional multicast capacity regions of such a wireless network. These inner and outer bounds differ only by a factor $O(\log (n))$, yielding a fairly tight scaling characterization of the entire regions. The communication schemes achieving the inner bounds use interference alignment as a central technique and are, at least conceptually, surprisingly simple.
\end{abstract}

\section{Index Terms}

Capacity scaling, interference alignment, multicast, multicommodity flow, opportunistic communication, wireless networks.

\section{INTRODUCTION}

Interference alignment is a recently introduced technique to cope with the transmissions of interfering users in wireless systems see [1]-[3]. In this paper, we apply this technique to obtain fairly precise (up to $O(\log (n))$ factor) information-theoretic scaling results for the unicast and multicast capacity regions of dense wireless networks.

\section{A. Related Work}

The study of scaling laws for wireless networks, describing the system performance in the limit of large number of users, was initiated by Gupta and Kumar in [4]. They analyzed a network scenario in which $n$ nodes are placed uniformly at random on a square of area one (called a dense network in the following) and are randomly paired into $n$ source-destination pairs with uniform rate requirement. Under a so-called protocol channel model, in which only point-to-point communication is allowed and interference is treated as noise, they showed that the largest uniformly achievable per-node rate scales as $\Theta\left(n^{-1 / 2}\right)$ up to a polylogarithmic factor in $n$. Achievability was shown using a multi-hop communication scheme combined with straight-line routing. Different constructions achieving slightly better scaling laws, i.e., improving the polylogarithmic factor in $n$, were subsequently presented in [5], [6].

These results are in some sense negative, in that they show that with current technology, captured by the protocol channel model assumption, the per-node rate in large wireless networks decreases with increasing network size even if the deployment area is kept constant. An immediate question is therefore if this negative result is due to the protocol channel model assumption or if there is a more fundamental reason for it. To address this question, several authors have considered an information-theoretic approach to the problem, in which the channel is simply assumed to be a Gaussian fading channel without any restrictions on the communication scheme [7]-[11]. We shall refer to this as the Gaussian fading channel model in the following. These works construct cooperative communication schemes and show that they can significantly outperform multi-hop communication in dense networks. In particular, Özgür et al. showed in [11] that in Gaussian fading dense wireless networks with randomly deployed nodes and random sourcedestination pairing, the maximal uniformly achievable per-node rate scales like $\Theta\left(n^{ \pm \varepsilon}\right)$ for any $\varepsilon>0$.

U. Niesen is with the Mathematics of Networks and Communications Research Department, Bell Labs, Alcatel-Lucent. Email: urs.niesen@alcatel-lucent.com

${ }^{1}$ The notation $\Theta\left(n^{ \pm \varepsilon}\right)$ is used to indicate that the maximal uniformly achievable per-node rate is upper bounded by $O\left(n^{\varepsilon}\right)$ and lower bounded by $\Omega\left(n^{-\varepsilon}\right)$. Similar expressions will be used throughout this section. 
In other words, in dense network 2 $^{2}$, cooperative communication can increase achievable rates to almost constant scaling in $n$-significantly improving the $\Theta\left(n^{-1 / 2}\right)$ scaling resulting from the protocol channel model assumption. The $\Theta\left(n^{ \pm \varepsilon}\right)$ scaling law was subsequently tightened to $n^{ \pm \Theta\left(\log ^{-1 / 2}(n)\right)}$ in [20], [21].

While these results removed the protocol channel model assumption made in [4], they kept the assumptions of random node placement and random source-destination pairing with uniform rate. Wireless networks with random node placement and arbitrary traffic pattern have been analyzed in [22], [23] for the protocol channel model and in [24] for the Gaussian fading channel model. On the other hand, wireless networks with arbitrary node placement and random source-destination pairing with uniform rate have been investigated in [25] for the protocol channel model and in [20] for the Gaussian fading channel model. While methods similar to the ones developed in [25] can also be used to analyze wireless networks with arbitrary node placement and arbitrary traffic pattern under the protocol channel model, the performance of such general networks under a Gaussian channel model (i.e., an information-theoretic characterization of achievable rates) is unknown.

Finally, it is worth mentioning [26], [27], which derive scaling laws for large dense interference networks. In particular, [27] considers a dense random node placement with random source-destination pairing. However, the model there is an interference channel as opposed to a wireless network as modeled in the works mentioned above. In other words, the source nodes cannot communicate with each other, and similarly the destination nodes cannot communicate with each other. This differs from the model adopted in this paper and the works surveyed so far, in which no such restrictions are imposed. For such interference networks, [27] derives the asymptotic sum-rate as the number of nodes in the network increases.

\section{B. Summary of Results}

In this paper, we consider the general problem of determining achievable rates in dense wireless networks with arbitrary node placement and arbitrary traffic pattern. We assume a Gaussian fading channel model, i.e., the analysis is information-theoretic, imposing no restrictions on the nature of communication schemes used. We analyze the $n \times n$-dimensional unicast capacity region $\Lambda^{\mathrm{UC}}(n) \subset \mathbb{R}_{+}^{n \times n}$, and the $n \times 2^{n}$ dimensional multicast capacity region $\Lambda^{\mathrm{MC}}(n) \subset \mathbb{R}_{+}^{n \times 2^{n}}$ of an arbitrary dense wireless network. $\Lambda^{\mathrm{UC}}(n)$ describes the collection of all achievable unicast traffic patterns (in which each message is to be sent to only one destination node), while $\Lambda^{\mathrm{MC}}(n)$ describes the collection of all achievable multicast traffic patterns (in which each message is to be sent to a set of destination nodes). We provide explicit approximations $\hat{\Lambda}^{\mathrm{UC}}(n)$ and $\hat{\Lambda}^{\mathrm{MC}}(n)$ of $\Lambda^{\mathrm{UC}}(n)$ and $\Lambda^{\mathrm{MC}}(n)$ in the sense that

$$
\begin{aligned}
& \hat{\Lambda}^{\mathrm{UC}}(n) \subset \Lambda^{\mathrm{UC}}(n) \subset K_{1} \log (n) \hat{\Lambda}^{\mathrm{UC}}(n), \\
& \hat{\Lambda}^{\mathrm{MC}}(n) \subset \Lambda^{\mathrm{MC}}(n) \subset K_{2} \log (n) \hat{\Lambda}^{\mathrm{MC}}(n),
\end{aligned}
$$

for constants $K_{1}, K_{2}$ not depending on $n$. In other words, $\hat{\Lambda}^{\mathrm{UC}}(n)$ and $\hat{\Lambda}^{\mathrm{MC}}(n)$ approximate the unicast and multicast capacity regions $\Lambda^{\mathrm{UC}}(n)$ and $\Lambda^{\mathrm{MC}}(n)$ up to a factor $O(\log (n))$. This provides tight scaling results for arbitrary traffic pattern and arbitrary node placement.

The results presented in this paper improve the known results in several respects. First, as already pointed out, they require no probabilistic modeling of the node placement or traffic pattern, but rather are valid for any node placement and any traffic pattern and include the results for random node placement and random source-destination pairing with uniform rate as a special case. Second, they provide informationtheoretic scaling results that are considerably tighter than the best previously known, namely up to a factor $O(\log (n))$ here as compared to $O\left(n^{\varepsilon}\right)$ in [11] and $n^{O\left(\log ^{-1 / 2}(n)\right)}$ in [20], [21]. Moreover, the results in this paper provide an explicit expression for the pre-constant in the $O(\log (n))$ term that is quite small, and

\footnotetext{
${ }^{2}$ We point out that the situation is quite different in extended networks, in which $n$ nodes are placed on a square of area $n$. Here network performance depends on the path-loss exponent $\alpha$, governing the speed of decay of signal power as a function of distance. For small $\alpha$, cooperative communication is order optimal, whereas for large $\alpha$, multi-hop communication is order optimal [11]-[19].
} 
hence these bounds yield good results also for small and moderate sized wireless networks. Third, the achievable scheme used to prove the inner bound in this paper is, at least conceptually, quite simple, in that the only cooperation needed between users is to perform interference alignment. This contrasts with the communication schemes achieving near linear scaling presented so far in the literature, which require hierarchical cooperation and are harder to analyze.

\section{Organization}

The remainder of this paper is organized as follows. Section 1 introduces the network model and notation. Section III presents the main results of this paper. Section IV describes the communication schemes used to prove achievability. Section $\mathrm{V}$ contains proofs, and Sections VI and VII contain discussions and concluding remarks.

\section{NeTwORK MODEL AND NotATiON}

Let

$$
A \triangleq[0,1]^{2}
$$

be a square of area one, and consider $n$ nodes $V(n) \subset A$ (with $|V(n)|=n$ ) placed in an arbitrary manner on $A$. Let $r_{u, v}$ be the Euclidean distance between nodes $u$ and $v$, and define

$$
r_{\min }(n) \triangleq n^{1 / 2} \min _{u \neq v} r_{u, v} .
$$

The minimum separation between nodes in the node placement $V(n)$ is then $r_{\min }(n) n^{-1 / 2}$. Note that $r_{\min }(n)=1$ for a grid graph, and $r_{\min }(n) \geq n^{-1}$ with high probability for $n$ nodes placed uniformly and independently at random on $A$. In general, we have

$$
r_{\min }(n) \leq 4 / \sqrt{\pi}<3
$$

and, while the results presented in this paper hold for any $r_{\min }(n)$, the case of interest is when $r_{\min }(n)$ decays at most polynomially with $n$, i.e., $r_{\min }(n) \geq n^{-\kappa}$ for some constant $\kappa \geq 0$. Note that we do not make any probabilistic assumptions on the node placement, but rather allow an arbitrary (deterministic) placement of nodes on $A$. In particular, the arbitrary node placement model adopted here contains the random node placement model as a special case. The arbitrary node placement model is, however, considerably more general since it allows for classes of node placements that only appear with vanishing probability under random node placement (e.g., node placements with large gaps or isolated nodes).

We assume the following complex baseband-equivalent channel model. The received signal $y_{v}[t]$ at node $v$ at time $t$ is given by

$$
y_{v}[t] \triangleq \sum_{u \neq v} h_{u, v}[t] x_{u}[t]+z_{v}[t]
$$

where $h_{u, v}[t]$ is the channel gain from node $u$ to node $v, x_{u}[t]$ is the signal sent by node $u$, and $z_{v}[t]$ is additive receiver noise at node $v$, all at time $t$. The additive noise components $\left\{z_{v}[t]\right\}_{v, t}$ are assumed to be independent and identically distributed (i.i.d.) circularly-symmetric complex Gaussian random variables with mean zero and variance one. The channel gain $h_{u, v}[t]$ has the form

$$
h_{u, v}[t] \triangleq r_{u, v}^{-\alpha / 2} \exp \left(\sqrt{-1} \theta_{u, v}[t]\right),
$$

where $\alpha \geq 2$ is the path-loss exponent. As a function of $u$ and $v$, the phase shifts $\left\{\theta_{u, v}[t]\right\}_{u, v}$ are assumed to be i.i.d. uniformly distributed over $[0,2 \pi)$. As a function of time $t$, we only assume that $\left\{\theta_{u, v}[t]\right\}_{t}$ varies in a stationary ergodic manner as a function of $t$ for every $u, v \in V(n)$. Note that the distances $r_{u, v}$ between the nodes do not change as a function of time and are assumed to be known throughout the network. The phase shifts $\left\{\theta_{u, v}[t]\right\}_{u, v}$ are assumed to be known at time $t$ at every node in the network. Together with the knowledge of the distances $\left\{r_{u, v}\right\}_{u, v}$, this implies that full causal channel state information (CSI) is 
available throughout the network. We impose a unit average power constraint on the transmitted signal $\left\{x_{u}[t]\right\}_{t}$ at every node $u$ in the network.

The phase-fading model (2) is adopted here for consistency with the capacity-scaling literature. All results presented in this paper can be extended to Rayleigh fading, see Section VI-C

A unicast traffic matrix $\lambda^{\mathrm{UC}} \in \mathbb{R}_{+}^{n \times n}$ associates with every node pair $(u, w) \in V(n) \times V(n)$ the rate $\lambda_{u, w}^{\mathrm{UC}}$ at which node $u$ wants to transmit a message to node $w$. The messages corresponding to distinct $(u, w)$ pairs are assumed to be independent. Note that we allow the same node $u$ to be source for several destinations $w$, and the same node $w$ to be destination for several sources $u$. The unicast capacity region $\Lambda^{\mathrm{UC}}(n) \subset \mathbb{R}_{+}^{n \times n}$ is the closure of the collection of all achievable unicast traffic matrices $\lambda^{\mathrm{UC}} \in \mathbb{R}_{+}^{n \times n}$. Knowledge of the unicast capacity region $\Lambda^{\mathrm{UC}}(n)$ provides hence information about the achievability of any unicast traffic matrix $\lambda^{\mathrm{UC}}$.

A multicast traffic matrix $\lambda^{\mathrm{MC}} \in \mathbb{R}_{+}^{n \times 2^{n}}$ associates with every pair of node $u \in V(n)$ and subset $W \subset V(n)$ the rate $\lambda_{u, W}^{\mathrm{MC}}$ at which node $u$ wants to multicast a message to the nodes in $W$, i.e., every node $w \in W$ wants to receive the same message from $u$. The messages corresponding to distinct $(u, W)$ pairs are again assumed to be independent. Note that we allow the same node $u$ to be source for several multicast groups $W$, and the same subset $W$ of nodes to be multicast group for several sources $u$. The multicast capacity region $\Lambda^{\mathrm{MC}}(n) \subset \mathbb{R}_{+}^{n \times 2^{n}}$ is the closure of the collection of all achievable multicast traffic matrices $\lambda^{\mathrm{MC}} \in \mathbb{R}_{+}^{n \times 2^{n}}$. Observe that unicast traffic is a special case of multicast traffic, and hence $\Lambda^{\mathrm{UC}}(n)$ is a $\mathbb{R}^{n \times n}$-dimensional "slice" of the $\mathbb{R}^{n \times 2^{n}}$-dimensional region $\Lambda^{\mathrm{MC}}(n)$.

The next example illustrates the definitions of unicast and multicast traffic.

Example 1. Consider $n=4$ and $V(n)=\left\{v_{i}\right\}_{i=1}^{4}$. Assume node $v_{1}$ wants to transmit a message $m_{1,2}$ to node $v_{2}$ at a rate of 1 bit per second, and a message $m_{1,3}$ to node $v_{3}$ at rate 2 bits per second. Node $v_{2}$ wants to transmit a message $m_{2,3}$ at rate 3 bits per second to node $v_{3}$. The messages $\left\{m_{1,2}, m_{1,3}, m_{2,3}\right\}$ are assumed to be independent. This traffic requirement can be described by a unicast traffic matrix $\lambda^{\mathrm{UC}} \in \mathbb{R}_{+}^{4 \times 4}$ with $\lambda_{v_{1}, v_{2}}^{\mathrm{UC} \triangleq 1} \triangleq \lambda_{v_{1}, v_{3}}^{\mathrm{UC}} \triangleq 2, \lambda_{v_{2}, v_{3}}^{\mathrm{UC}} \triangleq 3$, and $\lambda_{u, w}^{\mathrm{UC}} \triangleq 0$ for all other $(u, w)$ pairs. Note that node $v_{1}$ is source for $v_{2}$ and $v_{3}$, and that node $v_{3}$ is destination for $v_{1}$ and $v_{2}$. Note also that node $v_{4}$ is neither a source nor a destination for any communication pair, and can hence be understood as a helper node.

Assume now node $v_{1}$ wants to transmit the same message $m_{1,\{3,4\}}$ to both $v_{3}$ and $v_{4}$ at rate 1 bit per second, and a private message $m_{1,\{3\}}$ to only node $v_{3}$ at rate 2 bits per second. Moreover, node $v_{2}$ wants to transmit the same message $m_{2,\{3,4\}}$ to both $v_{3}$ and $v_{4}$ at rate 3 bits per second. The messages $\left\{m_{1,\{3,4\}}, m_{1,\{3\}}, m_{2,\{3,4\}}\right\}$ are assumed to be independent. This traffic requirement can be described by a multicast traffic matrix $\lambda^{\mathrm{MC}} \in \mathbb{R}_{+}^{4 \times 16}$ with $\lambda_{v_{1},\left\{v_{3}, v_{4}\right\}}^{\mathrm{MC}} \triangleq 1, \lambda_{v_{1},\left\{v_{3}\right\}}^{\mathrm{MC}} \triangleq 2, \lambda_{v_{2},\left\{v_{3}, v_{4}\right\}}^{\mathrm{MC}} \triangleq 3$, and $\lambda_{u, W}^{\mathrm{MC}} \triangleq 0$ for all other $(u, W)$ pairs. Note that $v_{1}$ is source for two multicast groups $\left\{v_{3}, v_{4}\right\}$ and $\left\{v_{3}\right\}$, and that $\left\{v_{3}, v_{4}\right\}$ is multicast group for two sources $v_{1}$ and $v_{2}$.

Throughout, we denote by $\log$ and $\ln$ the logarithms with respect to base 2 and $e$, respectively. To simplify notation, we suppress the dependence on $n$ within proofs whenever this dependence is clear from the context.

\section{MAIN RESULTS}

We now present the main results of this paper. Section $\amalg I-A$ provides a scaling characterization of the unicast capacity region $\Lambda^{\mathrm{UC}}(n)$, and Section $\amalg I I-B$ provides a scaling characterization of the multicast capacity region $\Lambda^{\mathrm{MC}}(n)$ of a dense wireless network. Section $\amalg$ II-C contains example scenarios illustrating applications of the main theorems.

\section{A. Unicast Traffic}

Define

$$
\hat{\Lambda}^{\mathrm{UC}}(n) \triangleq\left\{\lambda^{\mathrm{UC}} \in \mathbb{R}_{+}^{n \times n}: \sum_{w \neq u} \lambda_{u, w}^{\mathrm{UC}} \leq 1 \forall u \in V(n), \sum_{u \neq w} \lambda_{u, w}^{\mathrm{UC}} \leq 1 \forall w \in V(n)\right\} .
$$




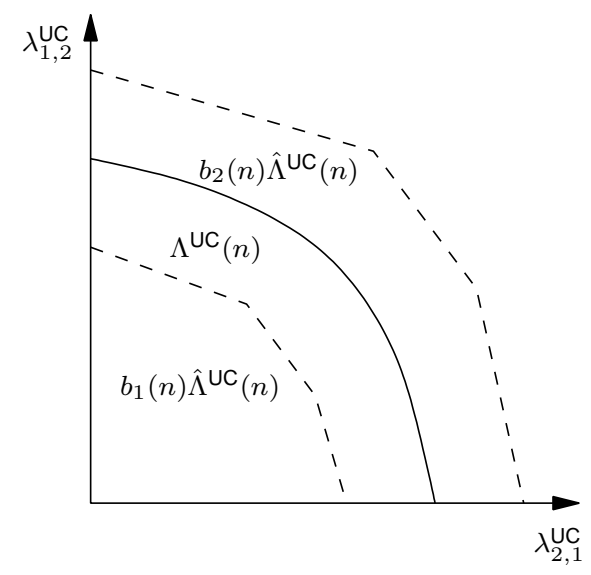

Fig. 1. The set $\hat{\Lambda}^{\mathrm{UC}}(n)$ approximates the unicast capacity region $\Lambda^{\mathrm{UC}}(n)$ of the wireless network in the sense that $b_{1}(n) \hat{\Lambda}^{\mathrm{UC}}(n)$, with $b_{1}(n)=2^{-\alpha / 2}$, provides an inner bound to $\Lambda^{\mathrm{UC}}(n)$ and $b_{2}(n) \hat{\Lambda}^{\mathrm{UC}}(n)$, with $b_{2}(n)=\log \left(n^{2+\alpha / 2} r_{\min }^{-\alpha}(n)\right)$, provides an outer bound to $\Lambda^{\mathrm{UC}}(n)$. The figure shows two dimensions (namely $\lambda_{1,2}^{\mathrm{UC}}$ and $\lambda_{2,1}^{\mathrm{UC}}$ ) of the $n \times n$-dimensional set $\Lambda^{\mathrm{UC}}(n)$.

$\hat{\Lambda}^{\mathrm{UC}}(n)$ is the collection of all unicast traffic matrices $\lambda^{\mathrm{UC}} \in \mathbb{R}_{+}^{n \times n}$ such that for every node $u$ in the network the total traffic

$$
\sum_{w \neq u} \lambda_{u, w}^{\mathrm{UC}}
$$

from $u$ is less than one, and such that for every node $w$ in the network the total traffic

$$
\sum_{u \neq w} \lambda_{u, w}^{\mathrm{UC}}
$$

to $w$ is less than one.

The next theorem shows that $\hat{\Lambda}^{\mathrm{UC}}(n)$ is a tight approximation of the unicast capacity region $\Lambda^{\mathrm{UC}}(n)$ of the wireless network.

Theorem 1. For all $\alpha \geq 2, n \geq 9$, and node placement $V(n)$ with minimum node separation $r_{\min }(n) n^{-1 / 2}$,

$$
2^{-\alpha / 2} \hat{\Lambda}^{\mathrm{UC}}(n) \subset \Lambda^{\mathrm{UC}}(n) \subset \log \left(n^{2+\alpha / 2} r_{\min }^{-\alpha}(n)\right) \hat{\Lambda}^{\mathrm{UC}}(n) .
$$

Assuming that $r_{\min }(n)$ decays no faster than polynomial in $n$ (see the discussion in Section (II), Theorem 1 states that $\hat{\Lambda}^{\mathrm{UC}}(n)$ approximates $\Lambda^{\mathrm{UC}}(n)$ up to a factor $O(\log (n))$. In other words, $\hat{\Lambda}^{\mathrm{UC}}(n)$ provides a scaling characterization of the unicast capacity region $\Lambda^{\mathrm{UC}}(n)$. This scaling characterization is considerably more general than the standard scaling results, in that it holds for any node placement and provides information on the entire $n \times n$-dimensional unicast capacity region (see Fig. 1). In particular, define

$$
\rho_{\lambda}^{\star} \cup \mathrm{C}(n) \triangleq \max \left\{\rho: \rho \lambda^{\mathrm{UC}} \in \Lambda^{\mathrm{UC}}(n)\right\}
$$

to be the largest multiple $\rho$ such that $\rho \lambda^{\mathrm{UC}}$ is achievable. Then, for any arbitrary node placement $V(n)$ and arbitrary unicast traffic matrix $\lambda^{\mathrm{UC}} \in \mathbb{R}_{+}^{n \times n}$, Theorem 1 determines $\rho_{\lambda}^{\star} \cup \mathrm{C}(n)$ up to a multiplicative gap of order $O(\log (n))$ uniform in $\lambda^{\mathrm{UC}}$. This contrasts with the standard scaling results, which provide information on $\rho_{\lambda}^{\star}(n)$ only for a uniform random node placement $V(n)$ and a uniform random unicast traffic matrix $\lambda^{\cup C}$ (constructed by pairing nodes randomly into $n$ source-destination pairs with uniform rate).

Theorem 1 also reveals that the unicast capacity region of a dense wireless network has a rather simple structure in that it can be approximated up to a factor $O(\log (n))$ by an intersection of $2 n$ half-spaces. Each of these half-spaces corresponds to a cut in the wireless network, bounding the total rate across this cut. While there are $2^{n}$ such cuts in the network, Theorem 1 implies that only a small fraction of them are of asymptotic relevance. From the definition of $\hat{\Lambda}^{\mathrm{UC}}(n)$, these are precisely the cuts involving just a single node (with traffic flowing either into or out of that node). 


\section{B. Multicast Traffic}

Let

$$
\hat{\Lambda}^{\mathrm{MC}}(n) \triangleq\left\{\lambda^{\mathrm{MC}} \in \mathbb{R}_{+}^{n \times 2^{n}}: \sum_{\substack{W \subset V(n): \\ W \backslash\{u\} \neq \emptyset}} \lambda_{u, W}^{\mathrm{MC}} \leq 1 \forall u \in V(n), \sum_{u \neq w} \sum_{\substack{W \subset V(n): \\ w \in W}} \lambda_{u, W}^{\mathrm{MC}} \leq 1 \forall w \in V(n)\right\} .
$$

Similarly to $\hat{\Lambda}^{\mathrm{UC}}(n)$ defined in Section $\amalg$ III-A, the region $\hat{\Lambda}^{\mathrm{MC}}(n)$ is the collection of multicast traffic matrices $\lambda^{\mathrm{MC}} \in \mathbb{R}_{+}^{n \times 2^{n}}$ such that for every node $u$ in the network the total traffic

$$
\sum_{\substack{W \subset V(n): \\ W \backslash\{u\} \neq \emptyset}} \lambda_{u, W}^{\mathrm{MC}}
$$

from $u$ is less than one, and such that for every node $w$ in the network the total traffic

$$
\sum_{u \neq w} \sum_{\substack{W \subset V(n): \\ w \in W}} \lambda_{u, W}^{\mathrm{MC}}
$$

to $w$ is less than one.

The next theorem shows that $\hat{\Lambda}^{\mathrm{MC}}(n)$ is a tight approximation of the multicast capacity region $\Lambda^{\mathrm{MC}}(n)$ of the wireless network.

Theorem 2. For all $\alpha \geq 2, n \geq 9$, and node placement $V(n)$ with minimum node separation $r_{\min }(n) n^{-1 / 2}$,

$$
2^{-1-\alpha / 2} \hat{\Lambda}^{\mathrm{MC}}(n) \subset \Lambda^{\mathrm{MC}}(n) \subset \log \left(n^{2+\alpha / 2} r_{\min }^{-\alpha}(n)\right) \hat{\Lambda}^{\mathrm{MC}}(n) .
$$

Assuming as before that $r_{\min }(n)$ decays no faster than polynomial in $n$, Theorem 2 asserts that $\hat{\Lambda}^{\mathrm{MC}}(n)$ approximates $\Lambda^{\mathrm{MC}}(n)$ up to a factor $O(\log (n))$. In other words, as in the unicast case, we obtain a scaling characterization of the multicast capacity region $\Lambda^{\mathrm{MC}}(n)$. Again, this scaling characterization is considerably more general than standard scaling results, in that it holds for any node placement and provides information about the entire $n \times 2^{n}$-dimensional multicast capacity region $\Lambda^{\mathrm{MC}}(n)$. Define, as for unicast traffic matrices,

$$
\rho_{\lambda}^{\star} \mathrm{MC}(n) \triangleq \max \left\{\rho: \rho \lambda^{\mathrm{MC}} \in \Lambda^{\mathrm{MC}}(n)\right\}
$$

to be the largest multiple $\rho$ such that $\rho \lambda^{\mathrm{MC}}$ is achievable. Then Theorem 2 allows, for any arbitrary node placement $V(n)$ and arbitrary multicast traffic matrix $\lambda^{\mathrm{MC}} \in \mathbb{R}_{+}^{n \times 2^{n}}$, to determine $\rho_{\lambda \mathrm{MC}}^{\star}(n)$ up to a multiplicative gap of order $O(\log (n))$ uniform in $\lambda^{\mathrm{MC}}$. In particular, no probabilistic assumptions about the structure of $V(n)$ or $\lambda^{\mathrm{MC}}$ are necessary.

As with $\Lambda^{\mathrm{UC}}(n)$, Theorem 2 implies that the multicast capacity region of a dense wireless network is approximated up to a factor $O(\log (n))$ by an intersection of $2 n$ half spaces. In other words, we are approximating a region of dimension $n \times 2^{n}$ (i.e., exponentially big in $n$ ) through only a linear number of inequalities. As in the case of unicast traffic, each of these inequalities corresponds to a cut in the wireless network, and it is again the cuts involving just a single node that are asymptotically relevant.

\section{Examples}

This section contains several examples illustrating various aspects of the capacity regions $\Lambda^{\mathrm{UC}}(n), \Lambda^{\mathrm{MC}}(n)$ and their approximations $\hat{\Lambda}^{\mathrm{UC}}(n), \hat{\Lambda}^{\mathrm{MC}}(n)$. Example 2 compares the scaling laws obtained in this paper with the ones obtained using hierarchical cooperation as proposed in [11]. Example 3 discusses symmetry properties of $\Lambda^{\mathrm{UC}}(n)$ and $\Lambda^{\mathrm{MC}}(n)$. Example 4 provides a traffic pattern showing that the outer bounds in Theorems 1 and 2 are tight up to a constant factor. 


\section{Example 2. (Random source-destination pairing)}

Consider a random node placement $V(n)$ with every node placed independently and uniformly at random on $A$. Assume we pair each node $u \in V(n)$ with a node $w \in V(n) \backslash\{u\}$ chosen independently and uniformly at random. Denote by $\left\{u_{i}, w_{i}\right\}$ the resulting $n$ source-destination pairs. Note that each node is source exactly once and destination on average once. Each source $u_{i}$ wants to transmit an independent message to $w_{i}$ at rate $\rho(n)$ (depending on $n$, but not on $i$ ). The question is to determine $\rho^{\star}(n)$, the largest achievable value of $\rho(n)$. This question was considered in [11], where it was shown that, with probability $1-o(1)$ as $n \rightarrow \infty$ and for every $\varepsilon>0$,

$$
\Omega\left(n^{-\varepsilon}\right) \leq \rho^{\star}(n) \leq O\left(n^{\varepsilon}\right) .
$$

The lower bound is achieved by a hierarchical cooperation scheme, and we denote its rate by $\rho_{\mathrm{HC}}(n)$.

We now show that using the results presented in this paper these bounds on $\rho^{\star}(n)$ can be significantly sharpened. Set $\lambda_{u_{i}, w_{i}}^{\mathrm{UC}} \triangleq 1$ for $i \in\{1, \ldots, n\}$ and $\lambda_{u, w}^{\mathrm{UC}} \triangleq 0$, for all other entries of $\lambda^{\mathrm{UC}} \cdot \rho^{\star}(n)$ is then given by

$$
\rho^{\star}(n)=\max \left\{\rho: \rho \lambda^{\mathrm{UC}} \in \Lambda^{\mathrm{UC}}(n)\right\} .
$$

Setting

$$
\hat{\rho}^{\star}(n) \triangleq \max \left\{\hat{\rho}: \hat{\rho} \lambda^{\mathrm{UC}} \in \hat{\Lambda}^{\mathrm{UC}}(n)\right\},
$$

we obtain from Theorem 1 that

$$
2^{-\alpha / 2} \hat{\rho}^{\star}(n) \leq \rho^{\star}(n) \leq \log \left(n^{2+\alpha / 2} r_{\min }^{-\alpha}(n)\right) \hat{\rho}^{\star}(n) .
$$

It remains to evaluate $\hat{\rho}^{\star}(n)$. By construction of $\lambda^{\mathrm{UC}}$, we have

$$
\max _{u \in V(n)} \sum_{w \neq u} \lambda_{u, w}^{\mathrm{UC}}=1
$$

Moreover, by [28],

$$
\mathbb{P}\left(\frac{1}{2} \leq \frac{\ln \ln (n)}{\ln (n)} \max _{w \in V(n)} \sum_{u \neq w} \lambda_{u, w}^{\cup C} \leq 2\right) \geq 1-o(1) .
$$

Using the definition of $\hat{\Lambda}^{\mathrm{UC}}(n)$, this yields that

$$
\frac{\ln \ln (n)}{2 \ln (n)} \leq \hat{\rho}^{\star}(n) \leq \frac{2 \ln \ln (n)}{\ln (n)}
$$

with high probability.

Recall that the minimum distance between nodes is $r_{\min }(n) n^{-1 / 2}$, and that, for a random node placement, $r_{\min }(n) \geq n^{-1}$ with high probability as $n \rightarrow \infty$ (see, e.g., [11, Theorem 3.1]). Hence (5) and (6) show that that for random node placement and random source-destination pairing

$$
2^{-1-\alpha / 2} \frac{\ln \ln (n)}{\ln (n)} \leq \rho^{\star}(n) \leq(4+3 \alpha) \log (e) \ln \ln (n)
$$

with probability $1-o(1)$ as $n \rightarrow \infty$. The lower bound is achieved using a communication scheme presented in Section IV-B based on interference alignment, and we denote its rate by $\rho_{\mathrm{IA}}(n)$.

Comparing (7) and (4), we see that the scaling law obtained here is significantly sharper, namely up to a factor $O(\log (n))$ here as opposed to a factor $O\left(n^{\varepsilon}\right)$ for any $\varepsilon>0$ in [11]. Moreover, (7) provides good estimates for any value of $n$, whereas (4) is only valid for large values of $n$, with a pre-constant in $O\left(n^{\varepsilon}\right)$ that increases rapidly as $\varepsilon \rightarrow 0$ (see [21], [29] for a detailed discussion on the dependence of the pre-constant on $\varepsilon$ ). For a numerical example, Table \ compares per-node rates $\rho_{\mathrm{HC}}(n)$ of the hierarchical cooperation scheme of [11] (more precisely, an upper bound to it, with optimized parameters as analyzed in [21]) with the per-node rates $\rho_{\mathrm{IA}}(n)$ obtained through interference alignment as proposed in this paper. For the numerical example, we choose $\alpha=4$. 
TABLE I

COMPARISON OF $\rho_{\mathrm{HC}}(n)$ AND $\rho_{\mathrm{IA}}(n)$ (IN BITS PER CHANNEL USE) FROM EXAMPLE2,

\begin{tabular}{r|llll} 
& $n=10^{2}$ & $n=10^{3}$ & $n=10^{4}$ & $n=10^{5}$ \\
\hline$\rho_{\mathrm{HC}}(n)$ & 0.0017 & 0.00047 & 0.00017 & 0.000070 \\
$\rho_{\mathrm{IA}}(n)$ & 0.042 & 0.035 & 0.030 & 0.027
\end{tabular}

We point out that the per-node rate $\rho_{\mathrm{IA}}(n)$ decreases as the number of nodes $n$ increases only because of the random source-destination pairing. In fact, if the nodes $\left\{u_{i}, w_{i}\right\}$ are paired such that each node is source and destination exactly once, then the interference alignment based scheme achieves a per-node rate $\rho_{\mathrm{IA}} \geq 2^{-\alpha / 2}$, i.e., the per-node rate does not decay to zero as $n \rightarrow \infty$.

Example 3. (Symmetry of $\Lambda^{\mathrm{UC}}(n)$ and $\Lambda^{\mathrm{MC}}(n)$ )

Theorems 1 and 2 provide some insight into (approximate) symmetry properties of the unicast and multicast capacity regions $\Lambda^{\mathrm{UC}}(n)$ and $\Lambda^{\mathrm{MC}}(n)$. Indeed, their approximations $\hat{\Lambda}^{\mathrm{UC}}(n)$ and $\hat{\Lambda}^{\mathrm{MC}}(n)$ are invariant with respect to node positions (and hence, in particular, also invariant under permutation of nodes).

More precisely, consider a unicast traffic matrix $\lambda^{\mathrm{UC}} \in \mathbb{R}_{+}^{n \times n}$. For a permutation $\pi$ of the nodes $V(n)$ set

$$
\tilde{\lambda}_{u, w}^{\mathrm{UC}} \triangleq \lambda_{\pi(u), \pi(w)}^{\mathrm{UC}} .
$$

Then $\lambda^{\mathrm{UC}} \in \hat{\Lambda}^{\mathrm{UC}}(n)$ if and only if $\tilde{\lambda}^{\mathrm{UC}} \in \hat{\Lambda}^{\mathrm{UC}}(n)$. Hence Theorem 1 yields that if $\lambda^{\mathrm{UC}} \in \Lambda^{\mathrm{UC}}(n)$, then

$$
2^{-\alpha / 2} \log ^{-1}\left(n^{2+\alpha / 2} r_{\min }^{-\alpha}(n)\right) \tilde{\lambda}^{\mathrm{UC}} \in \Lambda^{\mathrm{UC}}(n) .
$$

Similarly, let $\lambda^{\mathrm{MC}} \in \mathbb{R}_{+}^{n \times 2^{n}}$ be a multicast traffic matrix, and define

$$
\tilde{\lambda}_{u, W}^{\mathrm{MC}} \triangleq \lambda_{\pi(u), \pi(W)}^{\mathrm{MC}},
$$

where, for $W \subset V(n), \pi(W) \triangleq\{\pi(w): w \in W\}$. Theorem 2 implies that if $\lambda^{\mathrm{MC}} \in \Lambda^{\mathrm{MC}}(n)$, then

$$
2^{-1-\alpha / 2} \log ^{-1}\left(n^{2+\alpha / 2} r_{\min }^{-\alpha}(n)\right) \tilde{\lambda}^{\mathrm{MC}} \in \Lambda^{\mathrm{MC}}(n) .
$$

In other words, the location of the nodes in a dense wireless network (with $r_{\min }(n)$ decaying at most polynomially in $n$ ) affects achievable rates at most up to a factor $O(\log (n))$. This contrasts with the behavior of extended wireless networks, where node locations crucially affect achievable rates [20].

Example 4. (Tightness of outer bounds)

We now argue that the outer bounds in Theorems 1 and 2 are tight up to a constant factor in the following sense. There exists a constant $K>0$ such that for every $n$ we can find traffic matrices $\lambda^{\mathrm{UC}}$ and $\lambda^{\mathrm{MC}}$ on the boundary of the outer bound in Theorems 1 and 2 such that $K \lambda^{\mathrm{UC}} \in \Lambda^{\mathrm{UC}}(n)$ and $K \lambda^{\mathrm{MC}} \in \Lambda^{\mathrm{MC}}(n)$. Or, more succinctly, there exists a constant $K>0$ such that

$$
\begin{aligned}
& \Lambda^{\mathrm{UC}}(n) \backslash K \log \left(n^{2+\alpha / 2} r_{\min }^{-\alpha}(n)\right) \hat{\Lambda}^{\mathrm{UC}}(n) \neq \emptyset, \\
& \Lambda^{\mathrm{MC}}(n) \backslash K \log \left(n^{2+\alpha / 2} r_{\min }^{-\alpha}(n)\right) \hat{\Lambda}^{\mathrm{MC}}(n) \neq \emptyset .
\end{aligned}
$$

This shows that the $O(\log (n))$ gap between the inner and outer bounds in Theorems 1 and 2 is due to the use of the interference alignment scheme to prove the inner bound, and that to further decrease this gap a different achievable scheme has to be considered. Throughout this example, we assume $r_{\min }(n)>n^{-\kappa}$ for some constant $\kappa \geq 0$.

Choose a node $w^{\star} \in V(n)$, and let, for each $u, w \in V(n)$,

$$
\lambda_{u, w}^{\mathrm{UC}} \triangleq \begin{cases}\frac{1}{n-1} & \text { if } w=w^{\star} \\ 0 & \text { otherwise }\end{cases}
$$


Note that $\lambda^{\mathrm{UC}} \in \hat{\Lambda}^{\mathrm{UC}}(n)$. Under this traffic matrix $\lambda^{\mathrm{UC}}$, each node $u \in V(n)$ has an independent message for a common destination node $w^{\star}$.

If we ignore the received signals at all nodes $v \neq w^{\star}$ and transmit no signal at $w^{\star}$, we transform the wireless network into a multiple access channel with $n-1$ users. Since $r_{u, w^{\star}} \leq \sqrt{2}$ for any $u \in V(n)$, each node $u \in V(n) \backslash\left\{w^{\star}\right\}$ can reduce its power such that the received power at node $w^{\star}$ is equal to $2^{-\alpha / 2}$. In this symmetric setting, the equal rate point of the capacity region of the multiple access channel has maximal sum rate, and hence each node $u \in V(n) \backslash\left\{w^{\star}\right\}$ can reliably transmit its message to $w^{\star}$ at a per-node rate of

$$
\begin{aligned}
\frac{1}{n-1} \log \left(1+(n-1) 2^{-\alpha / 2}\right) & \geq \frac{1}{n-1} \log \left(n 2^{-\alpha / 2}\right) \\
& =\frac{1}{n-1}\left(1-\frac{\alpha}{2 \log (n)}\right) \log (n)
\end{aligned}
$$

Thus, for $n>2^{\alpha}$,

$$
\frac{1}{2} \log (n) \lambda^{\mathrm{UC}} \in \Lambda^{\mathrm{UC}}(n)
$$

On the other hand, using the assumption $r_{\min }(n)>n^{-\kappa}$,

$$
\log \left(n^{2+\alpha / 2} r_{\min }^{-\alpha}(n)\right)<(2+\alpha(1 / 2+\kappa)) \log (n),
$$

and hence

$$
(2+\alpha(1 / 2+\kappa)) \log (n) \lambda^{\mathrm{UC}} \notin \log \left(n^{2+\alpha / 2} r_{\min }^{-\alpha}(n)\right) \hat{\Lambda}^{\mathrm{UC}}(n)
$$

Therefore, setting

$$
K \triangleq(4+\alpha(1+2 \kappa))^{-1}>0
$$

we obtain from (8) and (9) that

$$
\Lambda^{\mathrm{UC}}(n) \backslash K \log \left(n^{2+\alpha / 2} r_{\min }^{-\alpha}(n)\right) \hat{\Lambda}^{\mathrm{UC}}(n) \neq \emptyset
$$

In words, at least along one direction in $\mathbb{R}^{n \times n}$, the outer bound in Theorem 1 is loose by at most a constant factor.

Since $\Lambda^{\mathrm{UC}}(n)$ is a $n \times n$-dimensional "slice" of the $n \times 2^{n}$-dimensional region $\Lambda^{\mathrm{MC}}(n)$, the same result follows for $\Lambda^{\mathrm{MC}}(n)$ as well.

\section{Communication Schemes}

This section describes the communication schemes achieving the inner bounds in Theorems 11 and 2 Both schemes use the idea of interference alignment as a building block, which is recalled in Section IV-A. The communication scheme for unicast traffic is introduced in Section IV-B and the scheme for multicast traffic in Section IV-C.

\section{A. Interference Alignment}

Interference alignment is a technique introduced recently in [1], [2]. The technique is best illustrated with an example taken from [3]. Assume we pair the nodes $V(n)$ into source-destination pairs $\left\{u_{i}, w_{i}\right\}_{i=1}^{n}$ such that each node in $V(n)$ is source and destination exactly once. Consider the channel gains $\left\{h_{u_{i}, w_{j}}\left[t_{1}\right]\right\}_{i, j}$ and $\left\{h_{u_{i}, w_{j}}\left[t_{2}\right]\right\}_{i, j}$ for two different times $t_{1}$ and $t_{2}$. Assume we could choose $t_{1}$ and $t_{2}$ such that $h_{u_{i}, w_{i}}\left[t_{1}\right]=$ $h_{u_{i}, w_{i}}\left[t_{2}\right]$ and $h_{u_{i}, w_{j}}\left[t_{1}\right]=-h_{u_{i}, w_{j}}\left[t_{2}\right]$ for all $i \neq j$. By adding up the received symbols $y_{w_{i}}\left[t_{1}\right]$ and $y_{w_{i}}\left[t_{2}\right]$, destination node $w_{i}$ obtains

$$
y_{w_{i}}\left[t_{1}\right]+y_{w_{i}}\left[t_{2}\right]=h_{u_{i}, w_{i}}\left[t_{1}\right]\left(x_{u_{i}}\left[t_{1}\right]+x_{u_{i}}\left[t_{2}\right]\right)+z_{w_{i}}\left[t_{1}\right]+z_{w_{i}}\left[t_{2}\right] .
$$


Thus, by sending the same symbol twice (i.e., $x_{u_{i}}\left[t_{1}\right]=x_{u_{i}}\left[t_{2}\right]$ ), every source node $u_{i}$ is able to communicate with its destination node $w_{i}$ at essentially half the rate possible without any interference from other nodes.

Using this idea and the symmetry and ergodicity of the distribution of the channel gains, the following result is shown in [3].

Theorem 3. For any source-destination pairing $\left\{u_{i}, w_{i},\right\}_{i=1}^{n}$ such that $u_{i} \neq u_{j}$ and $w_{i} \neq w_{j}$ for $i \neq j$, the rates

$$
\lambda_{u_{i}, w_{j}}^{\mathrm{UC}}= \begin{cases}\frac{1}{2} \log \left(1+2\left|h_{u_{i}, w_{i}}\right|^{2}\right) & \text { if } i=j, \\ 0 & \text { otherwise }\end{cases}
$$

are achievable, i.e., $\lambda^{\mathrm{UC}} \in \Lambda^{\mathrm{UC}}(n)$.

For a source-destination pairing $\left\{u_{i}, w_{i},\right\}_{i=1}^{n}$ as in Theorem 3 , construct a matrix $S \in \mathbb{R}_{+}^{n \times n}$ such that

$$
S_{u_{i}, w_{j}}= \begin{cases}1 & \text { if } i=j \\ 0 & \text { otherwise }\end{cases}
$$

Note that $S$ is a permutation matrix, and we will call such a traffic pattern a permutation traffic. Using $r_{u_{i}, w_{i}} \leq \sqrt{2}$ and $\alpha \geq 2$,

$$
\frac{1}{2} \log \left(1+2 r_{u_{i}, w_{i}}^{-\alpha}\right) \geq \frac{1}{2} \log \left(1+2^{1-\alpha / 2}\right) \geq 2^{-\alpha / 2},
$$

and hence Theorem 3 provides an achievable scheme showing that $2^{-\alpha / 2} S \in \Lambda^{\mathrm{UC}}(n)$. In other words, Theorem 3 shows that, for every permutation traffic, a per-node rate of $2^{-\alpha / 2}$ is achievable. In the next two sections, we will use this communication scheme for permutation traffic as a building block to construct communication schemes for general unicast and multicast traffic.

\section{B. Communication Scheme for Unicast Traffic}

Consider a general unicast traffic matrix $\lambda^{\mathrm{UC}} \in \mathbb{R}_{+}^{n \times n}$. If $\lambda^{\mathrm{UC}}$ happens to be a scalar multiple of a permutation matrix, then Theorem 3 provides us with an achievable scheme to transmit according to $\lambda^{\mathrm{UC}}$. In order to apply Theorem 3 for general $\lambda^{\mathrm{UC}}$, we need to schedule transmissions into several slots such that in each slot transmission occurs according to a permutation traffic. This transforms the original problem of communicating over a wireless network into a problem of scheduling over a switch with $n$ input and $n$ output ports and traffic requirement $\lambda^{\mathrm{UC}}$.

This problem has been widely studied in the literature. In particular, using a result from von Neumann [30] and Birkhoff [31] (see also [32] for the application to switches) it can be shown that for any $\lambda^{\mathrm{UC}} \in \hat{\Lambda}^{\mathrm{UC}}(n)$ there exist a collection of schedules $\left\{S_{i}\right\}$ (essentially permutation matrices, see the proof in Section $\nabla-A$ for the details) and nonnegative weights $\left\{\omega_{i}\right\}$ summing to one such that

$$
\sum_{i} \omega_{i} S_{i}=\lambda^{\mathrm{UC}}
$$

This suggests the following communication scheme. Split time into slots according to the weights $\left\{\omega_{i}\right\}$. In the slot corresponding to $\omega_{i}$, send traffic over the wireless network using interference alignment for the schedule $S_{i}$. In other words, we time share between the different schedules $\left\{S_{i}\right\}$ according to the weights $\left\{\omega_{i}\right\}$.

We analyze this communication scheme in more detail in Section $\mathrm{V}-\mathrm{A}$. In particular, we show that it achieves any point in $2^{-\alpha / 2} \hat{\Lambda}^{\mathrm{UC}}(n)$. Combined with a matching outer bound, we show that this scheme is optimal for any unicast traffic pattern up to a factor $2^{\alpha / 2} \log \left(n^{2+\alpha / 2} r_{\min }^{-\alpha}(n)\right)$.

Recall from Example 3 that the capacity region is approximately symmetric with respect to permutation of the traffic matrix. This implies that the rate achievable for any permutation traffic is approximately the same. While the decomposition of the traffic matrix $\lambda^{\mathrm{UC}}$ into schedules $\left\{S_{i}\right\}$ is not unique, this invariance 

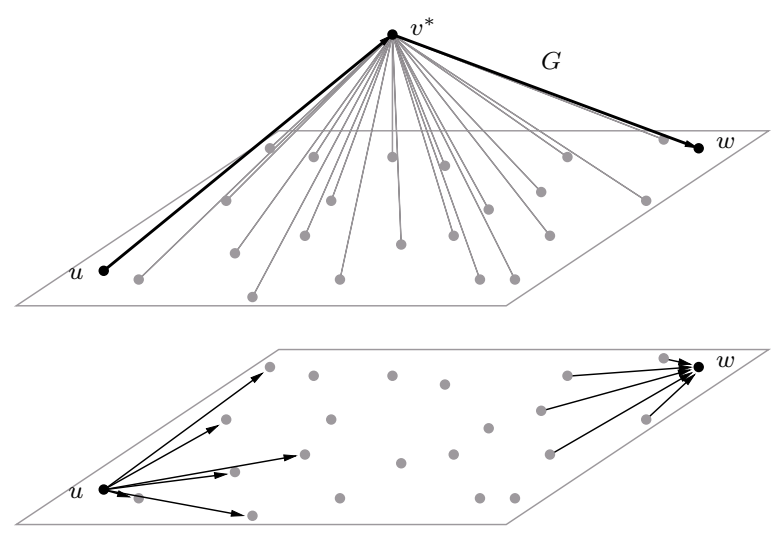

Fig. 2. Construction of the "star" graph $G$, and parts of the corresponding induced transmissions in the underlying wireless network for communication between $u$ and $w$.

suggests that it does not matter too much which decomposition is chosen. The situation is different for Rayleigh fading (as opposed to phase fading considered here), where different decompositions can be used for opportunistic communication. This approach is explored in detail in Section VI-C.

\section{Communication Scheme for Multicast Traffic}

We now turn to multicast traffic. Given the achievable scheme presented for unicast traffic in Section IV-B reducing the problem of communication over a wireless network to that of scheduling over a switch, it is tempting to try the same approach for multicast traffic as well. Unfortunately, scheduling of multicast traffic over switches is considerably more difficult than the corresponding unicast version (see, for example, [33] for converse results showing the infeasibility of multicast scheduling over switches with finite speedup). We therefore adopt a different approach here. The proposed communication scheme is reminiscent of the two-phase routing scheme of Valiant and Brebner [34].

Consider a source node $u \in V(n)$ that wants to multicast a message to destination group $W \subset V(n)$. The proposed communication scheme operates in two phases. In the first phase, the node $u$ splits its message into $n$ parts of equal length. It then sends one (distinct) part over the wireless network to each node in $V(n)$. Thus, after the first phase, each node in $V(n)$ has access to a distinct fraction $1 / n$ of the original message. In the second phase, each node in $V(n)$ sends its message parts to all the nodes in $W$. Thus, at the end of the second phase, each node in $W$ can reconstruct the entire message. All pairs $(u, W)$ operate simultaneously within each phase, and contention within the phases is resolved by appropriate scheduling (see the proof in Section $\mathrm{V}-\mathrm{B}$ for the details).

A different way to look at this proposed communication scheme is as follows. Consider the $n$ nodes in $V(n)$, and construct a graph $G=\left(V_{G}, E_{G}\right)$ with $V_{G} \triangleq V(n) \cup\left\{v^{\star}\right\}$ for some additional node $v^{\star} \notin V(n)$ and with $(u, v) \in E_{G}$ if either $u=v^{\star}$ or $v=v^{\star}$. In other words, $G$ is a "star" graph with central node $v^{\star}$ (see Fig. 2). We assign to each edge $e \in E_{G}$ an edge capacity of one. The proposed communication scheme for the wireless network can then be understood as a two layer architecture, consisting of a physical layer and a network layer. The physical layer implements the graph abstraction $G$, and the network layer routes data over $G$.

In Section $\mathrm{V}$-B, we show that the set of rates $\Lambda_{G}^{\mathrm{MC}}(n)$ that can be routed over $G$ contains $\hat{\Lambda}^{\mathrm{MC}}(n)$. We then argue that if $\lambda^{\mathrm{MC}} \in 2^{-1-\alpha / 2} \Lambda_{G}^{\mathrm{MC}}(n)$, then $\lambda^{\mathrm{MC}} \in \Lambda^{\mathrm{MC}}(n)$, i.e., if messages can be routed over the graph $G$ at rates $\lambda^{\mathrm{MC}}$, then almost the same rates are achievable in the wireless network. Combining this with a matching outer bound, we show that the proposed communication scheme is optimal for any multicast traffic pattern up to a factor $2^{1+\alpha / 2} \log \left(n^{2+\alpha / 2} r_{\min }^{-\alpha}(n)\right)$. 


\section{PROOFS}

This section contains the proofs of Theorem 1 (in Section $\nabla-A$ ) and Theorem 2 (in Section $\nabla-B$ ).

\section{A. Proof of Theorem 1}

We start with the proof of the outer bound in Theorem 11. For subsets $S_{1}, S_{2} \subset V, S_{1} \cap S_{2}=\emptyset$, denote by $C\left(S_{1}, S_{2}\right)$ the capacity of the multiple-input multiple-output (MIMO) channel between nodes in $S_{1}$ and nodes in $S_{2}$. Applying the cut-set bound [35, Theorem 14.10.1] to the sets $S_{1} \triangleq\{w\}^{c}, S_{2} \triangleq\{w\}$, we obtain

$$
\sum_{u \neq w} \lambda_{u, w}^{\mathrm{UC}} \leq C\left(\{w\}^{c},\{w\}\right)
$$

$C\left(\{w\}^{c},\{w\}\right)$ is upper bounded by relaxing the individual power constraints at each node to a sum-power constraint of $n-1$. This yields

$$
\begin{aligned}
C\left(\{w\}^{c},\{w\}\right) & \leq \log \left(1+(n-1) \sum_{u \neq w}\left|h_{u, w}\right|^{2}\right) \\
& =\log \left(1+(n-1) \sum_{u \neq w} r_{u, w}^{-\alpha}\right) .
\end{aligned}
$$

Since $r_{u, w} \geq n^{-1 / 2} r_{\min }$, we can continue this as

$$
\begin{aligned}
\log \left(1+(n-1) \sum_{u \neq w} r_{u, w}^{-\alpha}\right) & \leq \log \left(1+(n-1)^{2} n^{\alpha / 2} r_{\min }^{-\alpha}\right) \\
& \leq \log \left(1-n^{1+\alpha / 2} 3^{-\alpha}+n^{2+\alpha / 2} r_{\min }^{-\alpha}\right) \\
& \leq \log \left(n^{2+\alpha / 2} r_{\min }^{-\alpha}\right)
\end{aligned}
$$

where we have used that $r_{\min } \leq 3$ by (1) and that $n \geq 9$ by assumption. Hence

$$
\sum_{u \neq w} \lambda_{u, w}^{\mathrm{UC}} \leq \log \left(n^{2+\alpha / 2} r_{\min }^{-\alpha}\right)
$$

for all $w \in V$. Similarly,

$$
\sum_{w \neq u} \lambda_{u, w}^{\mathrm{UC}} \leq \log \left(n^{2+\alpha / 2} r_{\min }^{-\alpha}\right)
$$

for all $u \in V$.

Let $\lambda^{\mathrm{UC}} \in \Lambda^{\mathrm{UC}}$. From (10) and (11), we have that

$$
\lambda^{\mathrm{UC}} \in \log \left(n^{2+\alpha / 2} r_{\min }^{-\alpha}\right) \hat{\Lambda}^{\mathrm{UC}} .
$$

This implies

$$
\Lambda^{\mathrm{UC}} \subset \log \left(n^{2+\alpha / 2} r_{\min }^{-\alpha}\right) \hat{\Lambda}^{\mathrm{UC}}
$$

concluding the proof of the outer bound.

We continue with the proof of the inner bound. Consider a unicast traffic matrix $\lambda^{\mathrm{UC}} \in \hat{\Lambda}^{\mathrm{UC}}$. By definition of $\hat{\Lambda}^{\mathrm{UC}}$, this implies that

$$
\begin{aligned}
& \sum_{w \neq u} \lambda_{u, w}^{\mathrm{UC}} \leq 1 \forall u \in V, \\
& \sum_{u \neq w} \lambda_{u, w}^{\mathrm{UC}} \leq 1 \forall w \in V .
\end{aligned}
$$


Moreover, we can assume without loss of generality that $\lambda_{u, u}^{\mathrm{UC}}=0$ for all $u \in V$. Hence

$$
\begin{aligned}
& \sum_{w} \lambda_{u, w}^{\mathrm{UC}} \leq 1 \forall u \in V, \\
& \sum_{u} \lambda_{u, w}^{\mathrm{UC}} \leq 1 \forall w \in V .
\end{aligned}
$$

A matrix $\lambda^{\mathrm{UC}}$ satisfying the two conditions in (12) is called a doubly substochastic matrix. If $\lambda^{\mathrm{UC}}$ satisfies the conditions in (12) with equality, it is called a doubly stochastic matrix. Now, by [30, Lemma 1], for every doubly substochastic matrix $\lambda^{\mathrm{UC}} \in \mathbb{R}_{+}^{n \times n}$, there exists a doubly stochastic matrix $\tilde{\lambda}^{\mathrm{UC}} \in \mathbb{R}_{+}^{n \times n}$ such that $\lambda_{u, w}^{\mathrm{UC}} \leq \tilde{\lambda}_{u, w}^{\mathrm{UC}}$ for all $u, w \in V$. If we can show that $\tilde{\lambda}^{\mathrm{UC}}$ is achievable, then $\lambda^{\mathrm{UC}}$ is achievable as well. It suffices therefore to consider doubly stochastic traffic matrices, and we will assume in the following that $\lambda^{\mathrm{UC}}$ itself is doubly stochastic.

The set of doubly stochastic matrices of dimension $n \times n$ is convex and compact, and hence every matrix in this set can be written as a convex combination of its extreme points, see [36, Corollary 18.5.1]. Now, by Birkhoff's theorem [31, Theorem 1] (see, e.g., [37, Theorem 8.7.1] for a more recent reference), the extreme points of the set of doubly stochastic matrices are the permutation matrices. Hence there exists a collection of nonnegative weights $\left\{\omega_{i}\right\}$ summing to one and a collection of permutation matrices $\left\{S^{i}\right\}$ such that

$$
\sum_{i} \omega_{i} S^{i}=\lambda^{\mathrm{UC}}
$$

We time share between the different $\left\{S^{i}\right\}$ with weights given by $\omega_{i}$. Consider now transmission of messages according to one such permutation matrix $S^{i}$. Using the ergodic interference alignment strategy proposed in [3] (as summarized by Theorem 3 in Section IV-A]), each source-destination pair $(u, w)$ such that $S_{u, w}^{i}=1$ can simultaneously communicate at a per-node rate of

$$
\frac{1}{2} \log \left(1+2 r_{u, w}^{-\alpha}\right) \geq \frac{1}{2} \log \left(1+2^{1-\alpha / 2}\right) \geq 2^{-\alpha / 2},
$$

where we have used that $\alpha \geq 2$ in the second inequality. Thus, during the fraction of time corresponding to $\omega_{i}$, the nodes communicate at rates $2^{-\alpha / 2} S_{i}$.

With the time sharing described above and using (13), this shows that we can achieve

$$
\sum_{i} \omega_{i} 2^{-\alpha / 2} S_{i}=2^{-\alpha / 2} \lambda^{\mathrm{UC}}
$$

Therefore $2^{-\alpha / 2} \lambda^{\mathrm{UC}} \in \Lambda^{\mathrm{UC}}$, which implies

$$
2^{-\alpha / 2} \hat{\Lambda}^{\mathrm{UC}} \subset \Lambda^{\mathrm{UC}}
$$

proving the inner bound.

\section{B. Proof of Theorem 2}

We first prove the outer bound. Assume $\lambda^{\mathrm{MC}} \in \Lambda^{\mathrm{MC}}$. Fix a node $u \in V$, and choose for every subset $W \subset V$ such that $W \backslash\{u\} \neq \emptyset$ a node $\tilde{w}(W) \in W \backslash\{u\}$. Construct the unicast traffic matrix

$$
\lambda_{u, w}^{\mathrm{UC}} \triangleq \sum_{\substack{W \subset V: \\ \tilde{w}(W)=w}} \lambda_{u, W}^{\mathrm{MC}},
$$


for all $w \in V$, and $\lambda_{\tilde{u}, w}^{\mathrm{UC}} \triangleq 0$ for $\tilde{u} \neq u$. Note that $\lambda^{\mathrm{MC}} \in \Lambda^{\mathrm{MC}}$ implies that $\lambda^{\mathrm{UC}} \in \Lambda^{\mathrm{UC}}$. Indeed, we can transmit unicast traffic according to $\lambda^{\mathrm{UC}}$ by using the scheme for the multicast traffic matrix $\lambda^{\mathrm{MC}}$ and simply discarding the delivered messages for subset $W$ at all nodes $W \backslash\{\tilde{w}(W)\}$. Applying Theorem 1 ,

$$
\begin{aligned}
\sum_{\substack{W \subset V: \\
W \backslash\{u\} \neq \emptyset}} \lambda_{u, W}^{\mathrm{MC}} & =\sum_{w \neq u} \sum_{\substack{W \subset V: \\
\tilde{w}(W)=w}} \lambda_{u, W}^{\mathrm{MC}} \\
& =\sum_{w \neq u} \lambda_{u, w}^{\mathrm{UC}} \\
& \leq \log \left(n^{2+\alpha / 2} r_{\min }^{-\alpha}\right) .
\end{aligned}
$$

Since the choice of $u$ was arbitrary, (14) holds for all $u \in V$.

Fix now a node $w \in V$, and construct the unicast traffic matrix

$$
\lambda_{u, w}^{\mathrm{UC}} \triangleq \sum_{\substack{W \subset V: \\ w \in W}} \lambda_{u, W}^{\mathrm{MC}}
$$

for all $u \in V$, and $\lambda_{u, \tilde{w}}^{\mathrm{UC}} \triangleq 0$ if $\tilde{w} \neq w$. As before, $\lambda^{\mathrm{MC}} \in \Lambda^{\mathrm{MC}}$ implies $\lambda^{\mathrm{UC}} \in \Lambda^{\mathrm{UC}}$. Hence, by Theorem 1

$$
\begin{aligned}
\sum_{u \neq w} \sum_{\substack{W \subset V: \\
w \in W}} \lambda_{u, W}^{\mathrm{MC}} & =\sum_{u \neq w} \lambda_{u, w}^{\mathrm{UC}} \\
& \leq \log \left(n^{2+\alpha / 2} r_{\text {min }}^{-\alpha}\right) .
\end{aligned}
$$

As before, the choice of $w$ was arbitrary, and hence (15) holds for all $w \in V$.

Combining (14) and (15) shows that $\lambda^{\mathrm{MC}} \in \Lambda^{\mathrm{MC}}$ implies

$$
\lambda^{\mathrm{MC}} \in \log \left(n^{2+\alpha / 2} r_{\min }^{-\alpha}\right) \hat{\Lambda}^{\mathrm{MC}} .
$$

Therefore

$$
\Lambda^{\mathrm{MC}} \subset \log \left(n^{2+\alpha / 2} r_{\min }^{-\alpha}\right) \hat{\Lambda}^{\mathrm{MC}}
$$

proving the outer bound.

We now prove the inner bound. We construct a graph $G=\left(V_{G}, E_{G}\right)$ such that $V \subset V_{G}$, i.e., the nodes in the wireless network are a subset of the nodes in the graph $G$. We show that if messages can be routed over $G$ at rates $\lambda^{\mathrm{MC}}$, then $2^{-1-\alpha / 2} \lambda^{\mathrm{MC}}$ is achievable over the wireless network. We then argue that $\hat{\Lambda}^{\mathrm{MC}}$ is a subset of the rates that are achievable by routing over $G$. Together this will yield the desired inner bound.

The graph $G$ is a directed capacitated "star" graph constructed as follows. Consider $V$ and pick an additional node $v^{\star} \notin V$. Set

$$
\begin{aligned}
& V_{G} \triangleq V \cup\left\{v^{\star}\right\}, \\
& E_{G} \triangleq\left\{(u, v): u=v^{\star} \text { or } v=v^{\star}\right\}
\end{aligned}
$$

(see Fig. 2 in Section IV-C). Assign an edge capacity $c_{e} \triangleq 1$ for all $e \in E_{G}$. Note that, since $V \subset V_{G}$, every multicast traffic matrix $\lambda^{\mathrm{MC}} \in \mathbb{R}_{+}^{n \times 2^{n}}$ for the wireless network is also a multicast traffic matrix for $G$ (involving only the subset $V \subset V_{G}$ of nodes as sources and destinations). Define $\Lambda_{G}^{\mathrm{MC}}$ as the collection of such multicast traffic matrices $\lambda^{\mathrm{MC}} \in \mathbb{R}_{+}^{n \times 2^{n}}$ that are achievable via routing over $G$.

We now argue that $\hat{\Lambda}^{\mathrm{MC}} \subset \Lambda_{G}^{\mathrm{MC}}$. Assume $\lambda^{\mathrm{MC}} \in \hat{\Lambda}^{\mathrm{MC}}$. Since

$$
\sum_{\substack{W \subset V: \\ W \backslash\{u\} \neq \emptyset}} \lambda_{u, W}^{\mathrm{MC}} \leq 1
$$


for every $u \in V$, we can route all traffic $\lambda_{u, W}^{\mathrm{MC}}$ that is requested at some node other than $u$ (i.e., such that $W \backslash\{u\} \neq \emptyset$ ) from $u$ to the central node $v^{\star}$. Since

$$
\sum_{u \neq w} \sum_{\substack{W \subset V: \\ w \in W}} \lambda_{u, W}^{\mathrm{MC}} \leq 1
$$

for all $w \in V$, we can route all traffic $\lambda_{u, W}^{\mathrm{MC}}$ that is requested at some node $w \subset W$ from the central node $v^{\star}$ to $w$. Together, this shows that $\lambda^{\mathrm{MC}} \in \Lambda_{G}^{\mathrm{MC}}$, and hence that

$$
\hat{\Lambda}^{\mathrm{MC}} \subset \Lambda_{G}^{\mathrm{MC}} .
$$

We next argue that $\Lambda_{G}^{\mathrm{MC}} \subset 2^{1+\alpha / 2} \Lambda^{\mathrm{MC}}$. To this end, we show that any operation on $G$ can be implemented in the wireless network at least at a factor $2^{-1-\alpha / 2}$ of the rate. For the implementation of $G$ in the wireless network, we time share between edges towards the central node $v^{\star}$ and from the central node. This leads to a factor 2 loss in rate. We implement all edges $\left\{\left(u, v^{\star}\right)\right\}_{u \in V}$ simultaneously, and similarly for all edges $\left\{v^{\star}, w\right\}_{w \in V}$.

Assume $\lambda^{\mathrm{MC}} \in \Lambda_{G}^{\mathrm{MC}}$, and consider an edge $\left(u, v^{\star}\right) \in E_{G}$. Routing a message from $u$ to $v^{\star}$ in $G$ is implemented as follows. Take the message at $u$ and split it into $n$ (distinct) parts of equal length. Each part is to be sent to one of the $n$ nodes in $V$. In other words, one part is kept at $u$, the other $n-1$ parts are sent over the wireless network. This procedure is followed for every message at every node $u \in V$. Note that the resulting traffic requirement is unicast, and denote it by $\tilde{\lambda}^{\mathrm{UC}}$. This unicast traffic matrix $\tilde{\lambda}^{\mathrm{UC}}$ is uniform, in the sense that each node $u \in V$ has traffic for every other node $w \in V$ at the same rate, i.e., $\tilde{\lambda}_{u, w}^{\mathrm{UC}}$ depends only on $u$ but is constant as a function of $w$. Moreover, since $\lambda^{\mathrm{MC}} \in \Lambda_{G}^{\mathrm{MC}}$,

$$
\sum_{w \neq u} \tilde{\lambda}_{u, w}^{\mathrm{UC}} \leq \sum_{\substack{W \subset V: \\ W \backslash\{u\} \neq \emptyset}} \lambda_{u, W}^{\mathrm{MC}} \leq 1
$$

for every $u \in V$, and where we have the first inequality (instead of equality) because one part of every message is kept at the source node $u$. Together, this implies that

$$
\tilde{\lambda}_{u, w}^{\mathrm{UC}} \leq 1 /(n-1)
$$

for all $u \neq w$, and we can assume without loss of generality that we have equality for every $u \neq w$. This traffic pattern can be expressed as a convex combination of $n-1$ permutation matrices, each of which can be implemented at a rate of at least $2^{-\alpha / 2}$ by using ergodic interference alignment [3] as in the proof of Theorem 1. Hence, accounting for the factor 2 loss due to time sharing, all edges $\left\{\left(u, v^{\star}\right)\right\}_{u \in V}$ in $G$ can be implemented simultaneously with a loss of at most a factor $2^{-1-\alpha / 2}$ in the wireless network.

Consider now an edge $\left(v^{\star}, w\right) \in E_{G}$. Recall that all messages originate at $V \subset V_{G}$, and hence to arrive at $v^{\star}$ in $G$ the message is distributed uniformly over the entire wireless network (as described in the previous paragraph). Routing a message from $v^{\star}$ to $w$ in $G$ can thus be implemented in the wireless network by transmitting all the message parts from nodes $u \neq w$ to $w$. We transmit this traffic as unicast traffic by duplicating all messages that are to be sent to more than one destination node. Denote again by $\tilde{\lambda}^{\mathrm{UC}}$ the resulting unicast traffic matrix. Since the messages are distributed uniformly, $\tilde{\lambda}_{u, w}^{\mathrm{UC}}$ depends only on $w$ but is constant as a function of $u$. Moreover, since $\lambda^{\mathrm{MC}} \in \Lambda_{G}^{\mathrm{MC}}$,

$$
\sum_{u \neq w} \tilde{\lambda}_{u, w}^{\mathrm{UC}} \leq \sum_{u \neq w} \sum_{\substack{W \subset V: \\ w \in W}} \lambda_{u, W}^{\mathrm{MC}} \leq 1
$$

for every $w \in V$. Together, this implies that

$$
\tilde{\lambda}_{u, w}^{\mathrm{UC}} \leq 1 /(n-1)
$$


for all $u \neq w$, and we can assume again that we have equality for all $u \neq w$. Expressing the resulting uniform traffic pattern as a convex combination of permutation matrices and using again ergodic interference alignment as in the previous paragraph shows that all edges $\left\{\left(v^{\star}, w\right)\right\}_{w \in V}$ in $G$ can be implemented simultaneously with a loss of at most a factor $2^{-1-\alpha / 2}$ in the wireless network.

Together this shows that if $\lambda^{\mathrm{MC}} \in \Lambda_{G}^{\mathrm{MC}}$ then

$$
2^{-1-\alpha / 2} \lambda^{\mathrm{MC}} \in \Lambda^{\mathrm{MC}},
$$

and thus

$$
\Lambda_{G}^{\mathrm{MC}} \subset 2^{1+\alpha / 2} \Lambda^{\mathrm{MC}}
$$

Combining (16) and (17) shows that

$$
2^{-1-\alpha / 2} \hat{\Lambda}^{\mathrm{MC}} \subset 2^{-1-\alpha / 2} \Lambda_{G}^{\mathrm{MC}} \subset \Lambda^{\mathrm{MC}},
$$

completing the proof of the inner bound.

\section{DISCUSSION}

Here we discuss several aspects of the proposed communication schemes. The dependence of the results on the network area $|A|$ is discussed in Section VI-A. Implementation issues are considered in Section VI-B. Extensions to Rayleigh fading (as opposed to phase fading) are discussed in Section VI-C

\section{A. Dependence on Network Area}

Throughout this paper, we have assumed a unit network area, i.e., $|A|=1$. The results presented generalize to networks of area

$$
|A|=|A(n)| \triangleq a(n)
$$

for general $a(n)$ depending on the number $n$ of nodes in the network. Define the minimum distance between nodes to be $r_{\min }(n) n^{-1 / 2} a^{1 / 2}(n)$; as before, we assume that $r_{\min }(n)$ decays at most polynomially in $n$. Then Theorem 1 takes the form

$$
\frac{1}{2} \log \left(1+2^{1-\alpha / 2} a^{-\alpha / 2}(n)\right) \hat{\Lambda}^{\mathrm{UC}}(n) \subset \Lambda^{\mathrm{UC}}(n) \subset \log \left(1+n^{2+\alpha / 2} r_{\min }^{-\alpha}(n) a^{-\alpha / 2}(n)\right) \hat{\Lambda}^{\mathrm{UC}}(n),
$$

and Theorem 2

$$
\frac{1}{4} \log \left(1+2^{1-\alpha / 2} a^{-\alpha / 2}(n)\right) \hat{\Lambda}^{\mathrm{MC}}(n) \subset \Lambda^{\mathrm{MC}}(n) \subset \log \left(1+n^{2+\alpha / 2} r_{\min }^{-\alpha}(n) a^{-\alpha / 2}(n)\right) \hat{\Lambda}^{\mathrm{MC}}(n) .
$$

Comparing the lower and upper bound in these two expressions, we see that they provide the correct scaling of the unicast and multicast capacity regions of the wireless network only if $a(n)=n^{o(1)}$, i.e., only if the region $A(n)$ grows slower than $n^{\beta}$ for any $\beta>0$. This is not surprising, since when $a(n)$ grows on the order of $n^{\beta}$ for $\beta>0$, the network is no longer solely interference limited, but rather also power limited. Under these conditions, interference alignment is not the appropriate communication strategy and some form of hierarchical cooperation [11], [20], [24], [38] or other form of cooperative communication will likely be necessary (at least in the low $\alpha$ regime).

\section{B. Implementing Interference Alignment}

While the ergodic interference scheme recalled in Section $I \mathrm{IV}-\mathrm{A}$ is conceptually simple, it suffers from very long coding delays for larger networks. Indeed, it is easily seen that the coding delay of the scheme grows at least like $\Omega\left(\exp \left(n^{2}\right)\right)$. To be implemented, coding schemes whose delay scales better with respect to the network size need to be used. Devising such coding schemes guaranteeing the same rates as ergodic interference alignment but with shorter delays would hence be of interest.

Similarly, the assumption of availability of full CSI at all nodes in the network is quite strong. Relaxing this assumption would be of interest. Some progress in this direction has been made in [39], in which a distributed algorithm for interference alignment using only local CSI is proposed. However, while this algorithm is observed to yield good results in some scenarios, no performance guarantee is given for general systems. 


\section{Rayleigh Fading}

Throughout this paper, we have assumed a simple phase-fading model described by (2). In this section, we discuss how the results presented for this model can be adapted to the case of Rayleigh fading. We will assume that the channel gains $\left\{h_{u, v}[t]\right\}$ are independent (but not identically distributed) as a function of $u, v$ and vary in a stationary ergodic manner in $t$. Each $h_{u, v}[t]$ is assumed to be circularly-symmetric complex Gaussian with mean zero and variance $r_{u, v}^{-\alpha}$. The realizations $\left\{h_{u, v}[t]\right\}_{u, v}$ are assumed to be known at time $t$ throughout the network, i.e., we assume again full CSI is available at all nodes.

Denote by $\Lambda^{\mathrm{MC}}(n) \subset \mathbb{R}_{+}^{n \times 2^{n}}$ the multicast capacity region, and define $\hat{\Lambda}^{\mathrm{MC}}(n)$ as in the phase-fading case [see (3)]. The next theorem approximates the multicast capacity region under Rayleigh fading.

Theorem 4. There exists $n_{0}$ such that for all $\alpha \geq 2, n \geq n_{0}$, and node placement $V(n)$ with minimum node separation $r_{\min }(n) n^{-1 / 2}$,

$$
\frac{1}{16}(\log \log (n)-\alpha / 2-\log \log (e)) \hat{\Lambda}^{\mathrm{MC}}(n) \subset \Lambda^{\mathrm{MC}}(n) \subset \log \left(4 n^{2+\alpha / 2} r_{\min }^{-\alpha}(n)\right) \hat{\Lambda}^{\mathrm{MC}}(n) .
$$

Comparing Theorem 4 for Rayleigh fading with the corresponding result Theorem 2 for phase fading, we see that the inner bound is enlarged by a factor of $\Theta(\log \log (n))$. This is the gain due to opportunistic communication enabled by the random amplitudes of the channel gains and the availability of full CSI. Achievability is based on opportunistic interference-alignment. Note that, since unicast traffic is a special case of multicast traffic, Theorem 4 also applies to $\Lambda^{\mathrm{UC}}(n)$.

Proof: We first prove the outer bound. We assume throughout that $n \geq 9$. Following the same steps as in the proof of Theorem 2, it suffices to upper bound the MIMO capacities $C\left(\{w\}^{c},\{w\}\right)$ and $C\left(\{u\},\{u\}^{c}\right)$. Relaxing again the individual power constraints to a sum-power constraint of $n-1$, and increasing the channel gains by multiplying each $h_{u, v}$ by

$$
\frac{r_{\min }^{-\alpha / 2} n^{\alpha / 4}}{r_{u, v}^{-\alpha / 2}} \geq 1,
$$

we obtain

$$
C\left(\{w\}^{c},\{w\}\right) \leq \max \mathbb{E}\left(\log \left(1+P(g) n^{\alpha / 2} r_{\min }^{-\alpha} g\right)\right)
$$

where

$$
g \triangleq \sum_{u \neq w}\left|r_{u, v}^{\alpha / 2} h_{u, w}\right|^{2}
$$

and where the maximization is over all power assignments $P(g)$ such that

$$
\mathbb{E}(P(g)) \leq n-1 \text {. }
$$

By [40], this maximization problem is solved by water filling. The optimal power allocation is

$$
P^{\star}(g)=\left(\frac{1}{g_{0}}-\frac{1}{n^{\alpha / 2} r_{\min }^{-\alpha} g}\right)^{+},
$$

with $g_{0}$ chosen such that

$$
\mathbb{E}\left(P^{\star}(g)\right)=n-1
$$

Noting that

$$
P^{\star}(g) \leq \frac{1}{g_{0}},
$$

we can upper bound

$$
\begin{aligned}
C\left(\{w\}^{c},\{w\}\right) & \leq \mathbb{E} \log \left(1+P^{\star}(g) n^{\alpha / 2} r_{\min }^{-\alpha} g\right) \\
& \leq \mathbb{E} \log \left(1+n^{\alpha / 2} r_{\min }^{-\alpha} g / g_{0}\right) \\
& \leq \log \left(1+n^{\alpha / 2} r_{\min }^{-\alpha} \mathbb{E}(g) / g_{0}\right) \\
& \leq \log \left(1+n^{1+\alpha / 2} r_{\min }^{-\alpha} / g_{0}\right),
\end{aligned}
$$


where we have used Jensen's inequality.

It remains to find a lower bound on $g_{0}$. From the power constraint,

$$
\begin{aligned}
n-1 & =\mathbb{E}\left(\frac{1}{g_{0}}-\frac{1}{n^{\alpha / 2} r_{\min }^{-\alpha} g}\right)^{+} \\
& =\int_{\gamma=n^{-\alpha / 2} r_{\min }^{\alpha} g_{0}}^{\infty} f_{g}(\gamma)\left(\frac{1}{g_{0}}-\frac{1}{n^{\alpha / 2} r_{\min }^{-\alpha} \gamma}\right) d \gamma \\
& \geq \frac{1}{2 g_{0}} \mathbb{P}\left(g \geq 2 n^{-\alpha / 2} r_{\min }^{\alpha} g_{0}\right) \\
& \geq \frac{1}{2 g_{0}} \mathbb{P}\left(g \geq 2 g_{0}\right),
\end{aligned}
$$

where we have used that $r_{\min } \leq 3$ by (11) and that $n \geq 9$ by assumption. The random variable $g$ is the sum of $n-1$ i.i.d. exponential random variables with mean one. Hence $g$ follows an Erlang distribution with density

$$
f_{g}(\gamma)=\frac{\gamma^{n-2} \exp (-\gamma)}{(n-2) !}
$$

and

$$
\mathbb{P}(g \geq \gamma)=\exp (-\gamma) \sum_{i=0}^{n-2} \frac{\gamma^{i}}{i !},
$$

both for $\gamma \geq 0$. From this,

$$
\begin{aligned}
\frac{1}{2 g_{0}} \mathbb{P}\left(g \geq 2 g_{0}\right) & =\frac{\exp \left(-2 g_{0}\right)}{2 g_{0}} \sum_{i=0}^{n-2} \frac{\left(2 g_{0}\right)^{i}}{i !} \\
& \geq \frac{\exp \left(-2 g_{0}\right)}{2 g_{0}} .
\end{aligned}
$$

Combined with (19), we obtain

$$
n-1 \geq \frac{1}{2 g_{0}} \exp \left(-2 g_{0}\right) .
$$

Assume $g_{0}<1 / 4(n-1)$; then

$$
\begin{aligned}
\frac{1}{2 g_{0}} \exp \left(-2 g_{0}\right) & >2(n-1) \exp (-1 / 2(n-1)) \\
& \geq 2(n-1) \exp (-1 / 2) \\
& \geq(n-1),
\end{aligned}
$$

contradicting (20). This shows that

$$
g_{0} \geq 1 / 4(n-1) .
$$

Combining (18) and (21),

$$
\begin{aligned}
C\left(\{w\}^{c},\{w\}\right) & \leq \log \left(1+4(n-1) n^{1+\alpha / 2} r_{\min }^{-\alpha}\right) \\
& \leq \log \left(4 n^{2+\alpha / 2} r_{\min }^{-\alpha}\right)
\end{aligned}
$$

for every $w \in V$, and for $n \geq 9$. Similarly

$$
C\left(\{u\},\{u\}^{c}\right) \leq \log \left(4 n^{2+\alpha / 2} r_{\min }^{-\alpha}\right)
$$

for every $u \in V$. This proves the outer bound on $\Lambda^{\mathrm{MC}}$. 
We continue with the proof of the inner bound. From the construction in the proof of Theorem 2 , it suffices to analyze communication according to the unicast traffic matrix $\lambda_{u, w}^{\mathrm{UC}}=\rho(n)$ for all $u \neq w$, for some $\rho(n)$ depending on $n$ but not on $u, w$. If this $\lambda^{\mathrm{UC}}$ is achievable for some $\rho(n)$, then

$$
\frac{n}{2} \rho(n) \hat{\Lambda}^{\mathrm{MC}} \subset \Lambda^{\mathrm{MC}}
$$

Construct an undirected graph $\tilde{G}[t]=\left(V_{\tilde{G}}[t], E_{\tilde{G}}[t]\right)$ as follows. The vertex set $V_{\tilde{G}}[t]$ is equal to the collection of nodes $V$ in the wireless network for every $t \in \mathbb{N}$. The edge $(u, v)$ is in $E_{\tilde{G}}[t]$ if

$$
\max \left\{\left|h_{u, v}[t]\right|^{2},\left|h_{v, u}[t]\right|^{2}\right\} \geq \ln (1 / p(n)) r_{u, v}^{-\alpha}
$$

with

$$
p(n) \triangleq 1 / \sqrt{n}
$$

Note that $\left|r_{u, v}^{\alpha / 2} h_{u, v}[t]\right|^{2}$ is exponentially distributed with unit mean, and hence

$$
\mathbb{P}\left(\left|h_{u, v}[t]\right|^{2} \geq \ln (1 / p(n)) r_{u, v}^{-\alpha}\right)=p(n)
$$

for every $u, v \in V$ with $u \neq v$. Thus $\tilde{G}[t]$ is a random graph with $n$ vertices and each edge present i.i.d. with probability $p(n)$.

The choice of $p(n)$ guarantees by [41, Theorem 7.14] that, with probability $1-o(1)$ as $n \rightarrow \infty$, the graph $\tilde{G}[t]$ has a matching covering at least $n-1$ vertices, i.e., there is at least one way to pair adjacent nodes in $\tilde{G}[t]$ such that that all (except for possibly one node if $n$ is odd) nodes in $V$ are member of exactly one pair 3 Choose $n_{0}$ such that this probability is at least $1 / 2$ for $n \geq n_{0}$. Whenever no such pairing exists, we do not communicate during that time slot; this yields a factor 2 loss in rate. Assume in the following that at least one such pairing exists. Pick one of the (possibly many) pairings at random. By construction of $E_{\tilde{G}}$, for every such pair $(u, v)$ either $\left|h_{u, v}[t]\right|^{2}$ or $\left|h_{v, u}[t]\right|^{2}$ is larger than

$$
\ln (n) r_{u, v}^{-\alpha} / 2 \geq 2^{-1-\alpha / 2} \ln (n) .
$$

For each pair $(u, v)$ choose $u$ as the source for $v$ if the magnitude of the channel gain from $u$ to $v$ is larger than from $v$ to $u$, and $v$ as a source for $u$ otherwise. During time $t$ we transmit according to this source-destination pairing at uniform rate $\tilde{\rho}[t]$.

Consider now all times $t$ that have resulted in the same source-destination pairing. Note that the construction of $\tilde{G}[t]$, and hence also the construction of the source-destination pairing, depends only on the magnitudes of the channel gains $h_{u, v}[t]$. Hence, conditioned on a particular realization of $\tilde{G}[t]$, the phases of the channel gains are still independently and uniformly distributed over $[0,2 \pi)$ for every $u, v \in V$. The fading, conditioned on the source-destination pairing resulting from $\tilde{G}[t]$, is therefore still circularly-symmetric, and we can hence apply ergodic interference alignment as in Theorem 3 to communicate at uniform rate

$$
\begin{aligned}
\tilde{\rho}[t] & \geq \frac{1}{2} \log \left(1+2^{-\alpha / 2} \ln (n)\right) \\
& \geq \frac{1}{2}(-\alpha / 2-\log \log (e)+\log \log (n)) .
\end{aligned}
$$

During each time $t$, at least $(n-1) / 2$ of the source nodes are transmitting at rate at least $\tilde{\rho}[t]$. By the random choice of source-destination pairing, over a long enough time period all node pairs $(u, w)$ communicate the same fraction of time. Accounting for the half of time slots during which the graph $\tilde{G}[t]$

\footnotetext{
${ }^{3}$ The precise threshold for the appearance of such a matching is, in fact, for $p(n)$ larger than $(\log (n)+\omega(1)) / n$. However, the weaker choice of $p(n)=1 / \sqrt{n}$ adopted here is sufficient for our purposes.
} 
has no valid pairing, this procedure achieves a rate between each of the $n(n-1)$ pairs $(u, w)$ with $u \neq w$ of at least

$$
\begin{aligned}
\rho(n) & \geq \frac{n-1}{4 n(n-1)}\left(\frac{1}{2}(-\alpha / 2-\log \log (e)+\log \log (n))\right) \\
& =\frac{1}{8 n}(-\alpha / 2-\log \log (e)+\log \log (n)) .
\end{aligned}
$$

By (22), this implies that for, $n \geq n_{0}$,

$$
\frac{1}{16}(-\alpha / 2-\log \log (e)+\log \log (n)) \hat{\Lambda}^{\mathrm{MC}}(n) \subset \Lambda^{\mathrm{MC}}(n),
$$

concluding the proof of achievability.

\section{CONCLUSIONS}

We presented inner and outer bounds on the $n \times n$-dimensional unicast capacity region $\Lambda^{\mathrm{UC}}(n)$ and the $n \times 2^{n}$-dimensional multicast capacity region $\Lambda^{\mathrm{MC}}(n)$ of a dense wireless network with $n$ nodes placed arbitrarily on a unit square. These bounds are tight up to a factor $O(\log (n))$ (with a pre-constant that is rather small), and hence they yield fairly tight scaling laws for achievable rates under any unicast or multicast traffic pattern and any node placement.

\section{ACKNOWLEDGMENTS}

I would like to thank B. Nazer for helpful discussions and the anonymous reviewers for their comments.

\section{REFERENCES}

[1] V. R. Cadambe and S. A. Jafar, "Interference alignment and degrees of freedom of the $K$-user interference channel," IEEE Trans. Inf. Theory, vol. 54, pp. 3425-3441, Aug. 2008.

[2] M. A. Maddah-Ali, A. S. Motahari, and A. K. Khandani, "Communication over MIMO X channels: Interference alignment, decomposition, and performance analysis," IEEE Trans. Inf. Theory, vol. 54, pp. 3457-3470, Aug. 2008.

[3] B. Nazer, M. Gastpar, S. A. Jafar, and S. Vishwanath, "Ergodic interference alignment," in Proc. IEEE ISIT, pp. 1769-1773, June 2009.

[4] P. Gupta and P. R. Kumar, "The capacity of wireless networks,” IEEE Trans. Inf. Theory, vol. 46, pp. 388-404, Mar. 2000.

[5] S. R. Kulkarni and P. Viswanath, "A deterministic approach to throughput scaling in wireless networks," IEEE Trans. Inf. Theory, vol. 50, pp. 1041-1049, June 2004.

[6] M. Franceschetti, O. Dousse, D. N. C. Tse, and P. Thiran, "Closing the gap in the capacity of wireless networks via percolation theory," IEEE Trans. Inf. Theory, vol. 53, pp. 1009-1018, Mar. 2007.

[7] P. Gupta and P. R. Kumar, "Towards and information theory of large networks: An achievable rate region," IEEE Trans. Inf. Theory, vol. 49, pp. 1877-1894, Aug. 2003.

[8] G. Kramer, M. Gastpar, and P. Gupta, "Cooperative strategies and capacity theorems for relay networks," IEEE Trans. Inf. Theory, vol. 51, pp. 3037-3063, Sept. 2005.

[9] L.-L. Xie and P. R. Kumar, "An achievable rate for the multiple-level relay channel," IEEE Trans. Inf. Theory, vol. 51, pp. 1348-1358, Apr. 2005.

[10] S. Aeron and V. Saligrama, "Wireless ad hoc networks: Strategies and scaling laws for the fixed SNR regime," IEEE Trans. Inf. Theory, vol. 53, pp. 2044-2059, June 2007.

[11] A. Özgür, O. Lévêque, and D. N. C. Tse, "Hierarchical cooperation achieves optimal capacity scaling in ad hoc networks," IEEE Trans. Inf. Theory, vol. 53, pp. 3549-3572, Oct. 2007.

[12] L.-L. Xie and P. R. Kumar, "A network information theory for wireless communication: Scaling laws and optimal operation," IEEE Trans. Inf. Theory, vol. 50, pp. 748-767, May 2004.

[13] A. Jovičić, P. Viswanath, and S. R. Kulkarni, "Upper bounds to transport capacity of wireless networks," IEEE Trans. Inf. Theory, vol. 50, pp. 2555-2565, Nov. 2004.

[14] F. Xue, L.-L. Xie, and P. R. Kumar, "The transport capacity of wireless networks over fading channels," IEEE Trans. Inf. Theory, vol. 51, pp. 834-847, Mar. 2005.

[15] O. Lévêque and İ. E. Telatar, "Information-theoretic upper bounds on the capacity of large extended ad hoc wireless networks," IEEE Trans. Inf. Theory, vol. 51, pp. 858-865, Mar. 2005.

[16] S. H. A. Ahmad, A. Jovičić, and P. Viswanath, "On outer bounds to the capacity region of wireless networks," IEEE Trans. Inf. Theory, vol. 52, pp. 2770-2776, June 2006.

[17] L.-L. Xie and P. R. Kumar, "On the path-loss attenuation regime for positive cost and linear scaling of the transport capacity in wireless networks," IEEE Trans. Inf. Theory, vol. 52, pp. 2313-2328, June 2006. 
[18] A. Özgür, O. Lévêque, and E. Preissmann, "Scaling laws for one- and two-dimensional random wireless networks in the low-attenuation regime," IEEE Trans. Inf. Theory, vol. 53, pp. 3573-3585, Oct. 2007.

[19] M. Franceschetti, M. D. Migliore, and P. Minero, "The capacity of wireless networks: Information theoretic and physical limits," IEEE Trans. Inf. Theory, vol. 55, pp. 3413-3424, Aug. 2009.

[20] U. Niesen, P. Gupta, and D. Shah, "On capacity scaling in arbitrary wireless networks," IEEE Trans. Inf. Theory, vol. 56, pp. 3959-3982, Sept. 2009.

[21] J. Ghaderi, L.-L. Xie, and X. Shen, "Hierarchical cooperation in ad hoc networks: Optimal clustering and achievable throughput," IEEE Trans. Inf. Theory, vol. 55, pp. 3425-3436, Aug. 2009.

[22] S. Subramanian, S. Shakkottai, and P. Gupta, "On optimal geographic routing in wireless networks with holes and non-uniform traffic," in Proc. IEEE INFOCOM, pp. 1328-1336, May 2007.

[23] S. Subramanian, S. Shakkottai, and P. Gupta, "Optimal geographic routing for wireless networks with near-arbitrary holes and traffic," in Proc. IEEE INFOCOM, pp. 2002-2010, Apr. 2008.

[24] U. Niesen, P. Gupta, and D. Shah, "The balanced unicast and multicast capacity regions of large wireless networks," IEEE Trans. Inf. Theory, vol. 56, pp. 2249-2271, May 2010.

[25] R. Madan, D. Shah, and O. Lévêque, "Product multicommodity flow in wireless networks," IEEE Trans. Inf. Theory, vol. 54, pp. 14601476, Apr. 2008.

[26] S. A. Jafar, "The ergodic capacity of interference networks," arXiv:0902.0838 [cs.IT], Feb. 2009.

[27] O. Johnson, M. Aldridge, and R. Piechocki, "Interference alignment-based sum capacity bounds for random dense Gaussian interference networks," arXiv:0907.5165[cs.IT], July 2009.

[28] M. Raab and A. Steger, "Balls into bins-a simple and tight analysis," in Proc. RANDOM, pp. 159-170, 1998.

[29] L.-L. Xie, "Information theoretic scaling laws for wireless networks," arXiv:0809.1205 [cs.IT], July 2009.

[30] J. von Neumann, "A certain zero-sum two-person game equivalent to the optimal assignment problem," in Contributions to the Theory of Games (H. W. Kuhn and A. W. Tucker, eds.), vol. 2, pp. 5-12, Princeton University Press, 1953.

[31] G. Birkhoff, "Tres observaciones sobre el algebra lineal," Univ. Nac. Tucumán Rev, vol. 5, pp. 147-151, 1946.

[32] C.-S. Chang, W.-J. Chen, and H.-Y. Huang, "Birkhoff-von Neumann input buffered crossbar switches," in Proc. IEEE INFOCOM, pp. 1614-1623, Mar. 2000.

[33] M. A. Marsan, A. Bianco, P. Giaccone, E. Leonardi, and F. Neri, "Multicast traffic in input-queued switches: Optimal scheduling and maximum throughput," IEEE/ACM Trans. Netw., vol. 11, pp. 465-477, June 2003.

[34] L. G. Valiant and G. J. Brebner, "Universal schemes for parallel communication," in Proc. ACM STOC, pp. 263-277, 1981.

[35] T. M. Cover and J. A. Thomas, Elements of Information Theory. Wiley, 1991.

[36] R. T. Rockafellar, Convex Analysis. Princeton University Press, 1996.

[37] R. A. Horn and C. R. Johnson, Matrix Analysis. Cambridge University Press, 1985.

[38] A. Özgür, R. Johari, D. Tse, and O. Lévêque, "Information theoretic operating regimes of large wireless networks," in Proc. IEEE ISIT, pp. 186-190, July 2008.

[39] K. Gomadam, V. R. Cadambe, and S. A. Jafar, "Approaching the capacity of wireless networks through distributed interference alignment," in Proc. IEEE GLOBECOM, pp. 1-6, May 2007.

[40] A. J. Goldsmith and P. P. Varaiya, "Capacity of fading channels with channel side information," IEEE Trans. Inf. Theory, vol. 43, pp. 1986-1992, Nov. 1997.

[41] B. Bollobás, Random Graphs. Cambridge University Press, second ed., 2001. 